Silvana Granado Noguera da Gama² Ana Paula EsteVes Pereira ${ }^{3}$

Paulo RoBerto Borges de SOUZA JUNIOR ${ }^{4}$

\title{
Adequação do ganho ponderal de gestantes em dois municípios do Estado do Rio de Janeiro (RJ), Brasil, 2008
}

\author{
Adequacy of weight gain in pregnant women from two municipalities \\ of Rio de Janeiro state (RJ), Brazil, 2008
}

Artigo Original

Palavras-chave
Gestantes
Ganho de peso/epidemiologia
Estado nutricional
Keywords
Pregnant women
Weight gain/epidemiology
Nutritional status

\section{Resumo}

OBJETIVO: Avaliar fatores sociodemográficos, comportamentais, reprodutivos e morbidades associados à inadequação do ganho de peso gestacional. MÉTODOS: Estudo de coorte, desenvolvido entre dezembro de 2007 e agosto de 2008, incluindo mulheres no primeiro trimestre de gestação, acompanhadas no pré-natal de unidades do Sistema Único de Saúde (SUS) nos municípios de Petrópolis e Queimados, estado do Rio de Janeiro. Foram excluídas as mulheres que residiam fora desses municípios, com gravidez múltipla, aborto e sem as informações necessárias para o cálculo do estado nutricional pré-gestacional e de ganho de peso materno. Tanto o estado nutricional pré-gestacional quanto o ganho de peso gestacional foram avaliados segundo o critério preconizado pelo Institute of Medicine. A análise estatística foi realizada por modelo de regressão logística multinomial. RESULTADOS: Foram incluídas 1.287 gestantes. Verificaram-se 26,6\% de sobrepeso ou obesidade pré-gestacional e 1 1,0\% de baixo peso. A inadequação no ganho de peso gestacional foi observada em $71,4 \%$ das gestantes, sendo que, 35,6\% ganharam peso insuficiente e 35,8\% acima do recomendado. Na análise multivariada, mulheres hipertensas $(O R=2,1 ;$ IC $95 \%$ 1,4-3, 1), com sobrepeso $(O R=2,5$; IC95\% 1,4-4,5), obesidade pré-gestacional $(O R=2,7$; IC95\% 1,8-3,9) e com maior nível de escolaridade tiveram mais chance de ganhar peso acima do recomendado. Por outro lado, o baixo pré-gestacional $\mid O R=0,6$; IC95\% 0,4-0,9) foi proteção para o ganho excessivo. CONCLUSÃO: $\bigcirc$ diagnóstico nutricional prégestacional e o monitoramento do ganho de peso durante a gestação devem ser ações instituídas efetivamente na rotina do profissional de saúde.

\section{Abstract}

PURPOSE: To evaluate sociodemographic, behavioral and reproductive factors and morbidities associated with inadequate weight gain during pregnancy. METHODS: Cohort study conducted from December 2007 to August 2008 with women in the first trimester of pregnancy looking for prenatal care in the Public Health System who lived in the cities of Petrópolis or Queimados, Rio de Janeiro state (Brazill). Women with multiple pregnancy, who had a miscarriage in the index pregnancy or who lacked information for the assessment of pregravid nutritional status or weight gain were excluded. Pregravid nutritional status and weight gain during pregnancy were determined according to the criterion established by the Institute of Medicine (IOM). Statistical analysis was performed using a multinomial logistic regression model. RESULTS: A total of 1,287 women were included in the study; $26.6 \%$ of them were overweight or obese while $11 \%$ were underweight. Inadequate weight gain during pregnancy was observed in $71.4 \%$ of pregnant women; $35.6 \%$ of them did not gain enough weight while $35.8 \%$ gained more weight than recommended by the $1 O M$. In the multivariate analysis, women with hypertension $(O R=2.1 ; 95 \% \mathrm{Cl} 1.4-3.1)$, pregravid overweight $(O R=2.5 ; 95 \% \mathrm{Cl}$ 1.4-4.5) or obesity $(O R=2.7 ; 95 \% \mathrm{Cl} 1.8-3.9)$ and who had a higher educational level were more likely to gain more weight than recommended, while pregravid underweight $(O R=0.6 ; 95 \% \mathrm{Cl} 0.4-0.9)$ represented a protection against excessive gain. CONCLUSION: Pregravid nutritional diagnosis and weight gain monitoring should be actions effectively instituted in the routine of health professionals.
Correspondêncio

Daniele Marano

Escola Nacional de Saúde Pública Sergio Arouca Rua Leopoldo Buhhões, 1.480, sola 808 - Manguinhos

(EP: 21041-210

Rio de Janeiro (RJ), Brasil

Recebido

$30 / 03 / 2012$

Aceito com modificacōes

$31 / 07 / 2012$
Trabalho realizado na Escola Nacional de Saúde Pública/Fundação Oswaldo Cruz - ENSP/FIOCRUZ - Rio de Janeiro (RJ), Brasil. IInstituto Fernandes Figueira/Fundação Oswaldo Cruz - IFF/FIOCRUZ - Rio de Janeiro (RI), Brasil,

2Departamento de Epidemiologia e Métodos Quantitativos em Saúde, Escola Nacional de Saúde Pública/Fundação Oswaldo Cruz ENSP/FIOCRUZ - Rio de Janeiro (RI), Brasil.

3Programa de Pós-graduação de Epidemiologia em Saúde Pública, Escola Nacional de Saúde Pública/Fundação Oswaldo Cruz ENSP/FIOCRUZ - Rio de Janeiro (RJ), Brasil.

4Departamento de Informação em Saúde, Instituto de Comunicação e Informação Científica e Tecnológica em Saúde/Fundação Oswaldo Cruz - ICICT/FIOCRUZ - Rio de Janeiro (R)), Brasil.

Conflito de interesses: não há. 


\section{Introdução}

A inadequação do ganho de peso durante a gestação tem sido apontada como fator de risco tanto para a mãe quanto para o concepto ${ }^{1,2}$. $\mathrm{O}$ ganho de peso aquém do recomendado pode acarretar restrição de crescimento intrauterino, parto prematuro, baixo peso ao nascer e aumento das taxas de morbimortalidade perinatal ${ }^{3-5}$. Enquanto o ganho excessivo está associado, no feto, a hemorragias, macrossomia, desproporção céfalo-pélvica, asfixia; e na mulher, diabetes mellitus gestacional, hipertensão arterial, pré-eclampsia, eclampsia ${ }^{6-9}$, maior retenção de peso pós-parto e aumento do risco de obesidade futura, contribuindo para a elevação da prevalência desse agravo ${ }^{10}$.

A grande variação no ganho de peso na gestação em mulheres saudáveis parece ser determinada por diferentes características maternas ${ }^{7,11}$. Gravidez na adolescência, baixo peso pré-gestacional, baixo nível de renda, hábito de fumar, consumo de álcool e de drogas na gestação contribuem para o ganho de peso gestacional insuficiente $^{3}$. Por outro lado, o ganho de peso excessivo pode ser determinado pela idade acima de 35 anos, sobrepeso ou obesidade pré-gestacional, ter companheiro, consumo de álcool na gestação e trabalhar fora de casa ${ }^{7,12,13}$.

Apesar de outros estudos já terem descrito os principais determinantes associados à inadequação ponderal gestacional, ressalta-se que alguns deles ainda não estão bem estabelecidos, sobretudo em relação à influência do nível de escolaridade ${ }^{7,8}$ e da classe econômica ${ }^{2}$. Além disso, um relevante fator, a insegurança alimentar, vem sendo, mais recentemente, incluída nesse debate.

Nesta perspectiva, o presente artigo teve como objetivo descrever os principais fatores associados à inadequação do ganho de peso em gestantes atendidas em estabelecimentos da rede do Sistema Único de Saúde (SUS) que prestam assistência pré-natal em dois municípios do estado do Rio de Janeiro.

\section{Métodos}

O presente trabalho consistiu em uma análise de dados coletados na primeira (gestação) e na segunda etapa (pós-parto) de seguimento de um estudo prospectivo com quatro pontos de observação (gestação, pós-parto, três e seis meses após o parto).

Foram incluídas 1.678 mulheres no primeiro trimestre de gestação, residentes nos municípios de Queimados e Petrópolis, estado do Rio de Janeiro, e atendidas nos serviços de pré-natal de 11 unidades de saúde da rede municipal do SUS, 8 no primeiro e 3 no segundo município, respectivamente.

Foram excluídas, da presente análise, 17 mulheres da amostra inicial devido à gestação múltipla,
62 que abortaram durante o estudo e 208 por não se disporem de informações necessárias para o cálculo do estado nutricional pré-gestacional e de ganho de peso materno. Além disso, houve a perda de seguimento de 104 mulheres da primeira para a segunda etapa, totalizando ao final uma amostra de 1.287 gestantes. Para a análise multivariada foram também excluídas 40 gestantes com relato de perda de peso e $21 \mathrm{com}$ ganho acima de $30 \mathrm{~kg}$, por constituírem potenciais erros de informação.

As unidades de saúde selecionadas foram identificadas no Cadastro Nacional de Estabelecimentos de Saúde ${ }^{14}$ e detinham $90 \%$ da cobertura do cuidado pré-natal do SUS em ambas as cidades. Os demais $10 \%$ não foram incluídos pela dificuldade logística em cobrir atendimentos esporádicos em diversas unidades da Estratégia de Saúde da Família.

A coleta de dados da primeira etapa, linha de base, ocorreu no período de dezembro de 2007 a agosto de 2008. O questionário foi aplicado por profissionais e estudantes da área da saúde previamente treinados e supervisionados por pesquisadores, após a consulta de pré-natal nas unidades de saúde. A entrevista pós-parto (segunda etapa) se deu no início do puerpério tendo como um dos objetivos a obtenção do peso da mulher ao final da gestação. Os instrumentos foram avaliados e pré-testados durante o treinamento de campo e no estudo piloto. Todos os questionários foram revisados e duplamente digitados.

Para avaliação antropométrica foi considerado o peso pré-gestacional relatado pela mulher na entrevista de linha de base. $\mathrm{Na}$ ausência desta informação, o dado foi coletado do cartão da gestante em consulta realizada até a $14^{a}$ semana de gestação. A altura, em centímetros, foi aferida pela equipe da pesquisa na unidade de saúde; utilizou-se estadiômetro em alumínio com escala de 2 metros e sensibilidade de $0,5 \mathrm{~cm}$ da balança mecânica da marca Filizola ${ }^{\circledR}$. Quando a unidade de saúde não dispunha do equipamento, a altura foi autorreferida, o que ocorreu em menos de $20 \%$ dos casos. Obteve-se o ganho de peso gestacional subtraindo-se o peso ao final da gravidez (segundo período de seguimento) pelo peso pré-gestacional (linha de base), considerando a idade gestacional (IG) estimada pelo exame de ultrassonografia. O peso ao final da gestação foi referido pela gestante e, na ausência desta informação, coletou-se do cartão até dez dias antes do parto.

O estado nutricional pré-gestacional foi classificado de acordo com o índice de massa corporal (IMC) pré-gestacional, com base nos novos pontos de corte propostos pelo Institute of Medicine ${ }^{15}$. Foram classificadas na faixa de baixo peso gestantes com IMC $<18,5 \mathrm{~kg} / \mathrm{m}^{2}$, eutróficas com 18,5 $<\mathrm{IMC}<24,9 \mathrm{~kg} / \mathrm{m}^{2}$, sobrepeso com 
$25<$ IMC $<29,9 \mathrm{~kg} / \mathrm{m}^{2}$ e obesas com IMC pré-gestacional $>30 \mathrm{~kg} / \mathrm{m}^{2}$.

Do mesmo modo, a variável dependente também foi baseada nas recomendações do IOM, que estabelecem limites de ganho de peso com base no estado nutricional pré-gestacional. Assim considera-se ganho de peso insuficiente: $<12,5 \mathrm{~kg}$ para mulheres com baixo peso; $<11,5 \mathrm{~kg}$ para as eutróficas; $<7,0 \mathrm{~kg}$ para mulheres com sobrepeso e $<5,0 \mathrm{~kg}$ para obesas. Ganho de peso adequado é definido como: 12,5 a $18,0 \mathrm{~kg}$ para mulheres com baixo peso; 11,5 a $16,0 \mathrm{~kg}$ para eutróficas; 7,0 a $11,5 \mathrm{~kg}$ para mulheres com sobrepeso e 5,0 a 9,0 kg para obesas. Finalmente, o ganho de peso é excessivo se $>18,0 \mathrm{~kg}$ para mulheres com baixo peso; $>16,0 \mathrm{~kg}$ para eutróficas; $>11,5 \mathrm{~kg}$ para mulheres com sobrepeso e $>9,0 \mathrm{~kg}$ para obesas. A faixa de ganho de peso adequado foi corrigida segundo a IG ao nascimento.

Os determinantes da inadequação do ganho de peso estudados foram (I) sociodemográficos: idade das gestantes em anos $(<19 ; 20-34 ;>35)$, escolaridade em anos de estudo, classe social (B/C; D/E), estado marital (vive com companheiro; não vive com companheiro), cor de pele segundo classificação da gestante (branca; preta; parda), trabalho durante a gravidez (não; sim); (II) estilo de vida: fumo (não; sim), álcool (não; sim); (III) adequação do pré-natal (mais que adequado; adequado; parcialmente adequado/inadequado); (V) doenças crônicas: hipertensão arterial pré-gestacional e gestacional (não; sim), diabetes mellitus pré-gestacional e gestacional (não; sim); (V) padrão alimentar: (alta quantidade de proteínas e frutas; tradicional; alta quantidade de carboidratos e gorduras; lanche); (VI) obstétricas: primiparidade (não; sim); (VII) estado nutricional pré-gestacional: (baixo peso; eutrófica; sobrepeso; obesa).

Para essa análise, optou-se agregar tanto a hipertensão arterial quanto a diabetes mellitus pré-gestacional com a gestacional pela dificuldade de distinguir o início dessas doenças. A variável "município de residência", Petrópolis ou Queimados, também foi incluída como variável independente por suas diferentes características socioeconômicas, tendo Queimados um dos piores índices de desenvolvimento humano (IDH) do estado $\left(73^{\text {a }}\right.$ posição entre as 91 cidades do estado do Rio de Janeiro, enquanto Petrópolis ocupa a $\left.7^{\mathrm{a}}\right)^{16}$. Por essa razão, Petrópolis foi utilizada como categoria de referência nos modelos de análise.

O critério adotado pela Associação Nacional de Empresas de Pesquisa (ANEP) foi empregado para a classificação econômica, com base nos bens da família, aos quais é aplicado um escore final definindo os grupos socioeconômicos: A (mais alto), B, C, D e E (mais baixo $)^{17}$. Nesse estudo as categorias foram agrupadas em B/C e D/E, não sendo incluída a classe social A, por nenhuma mulher ter sido classificada nessa categoria.

Para a avaliação da adequação do pré-natal foi utilizado o critério de Kotelchuck que se baseia em duas dimensões distintas: IG no início do pré-natal e número de consultas. O percentual de adequação do número de consultas é definido dividindo o número de consultas realizadas pelo número de consultas esperadas para a IG ao nascimento $\mathrm{x} 100$, definidas em quatro categorias de pré-natal: inadequado, parcialmente adequado, adequado e mais do que adequado ${ }^{18}$.

Para avaliar o consumo alimentar no último trimestre de gestação, foi aplicado o Questionário de Frequência Alimentar (QFA), baseado em um instrumento simplificado com 19 itens alimentares ${ }^{19}$. Adicionalmente, foram incluídos a este questionário 18 alimentos consumidos frequentemente pela população brasileira, especialmente fast foods e fontes de cafeína.

Foram definidos quatro padrões de consumo, agrupados da seguinte forma: alta quantidade de proteínas e frutas (composto por leite, iogurte, queijo, frutas e suco natural, biscoito sem recheio e carne de frango/ boi/peixe/fígado); tradicional (feijão, arroz, vegetais, pães, manteiga/margarina, açúcar); alta quantidade de carboidratos e gorduras (batata/aipim/inhame, macarrão, farinha/farofa/angu, carne de porco/salsicha/linguiça/ ovo, refrigerante/refresco, pizza/hambúrguer/pastel) e o lanche (biscoito recheado, biscoitos salgadinhos tipo Skiny ${ }^{\circledR} /$ Fofura $^{\circledR} /$ Fandangos $^{\circledR}$, chocolate e achocolatado).

O estudo foi aprovado pelo Comitê de Ética em Pesquisa da Escola Nacional de Saúde Pública (ENSP/ FIOCRUZ) em conformidade com as orientações da Declaração de Helsinki e da Comissão Nacional de Ética em Pesquisa (CONEP) e está de acordo com os princípios éticos de não maleficência, beneficência, justiça e autonomia, contidos na Resolução nº 196/96, do Conselho Nacional de Saúde ${ }^{20}$.

\section{Análise estatística}

Para avaliar a diferença bruta no ganho de peso durante a gestação foi realizada análise de variância (ANOVA). A normalidade da distribuição da variável "ganho de peso gestacional" foi analisada pelo teste de Kolmogorov-Smirnov no programa Statistical Package for the Social Sciences (SPSS) versão 17.0.

No modelo de regressão logística multinomial foram identificados os determinantes associados à inadequação do ganho de peso gestacional, tendo o ganho de peso adequado como categoria de referência.

Para definição do modelo final, foram inseridas as covariáveis com valor $\mathrm{p} \leq 0,20$ na análise bivariada. As análises foram realizadas no programa estatístico 
$\mathrm{R}$, versão 2.9.2 obtendo como medida de associação a Odds Ratio (OR). Com o objetivo de escolher a melhor estratégia de análise multivariada, foram ajustados dois modelos, a saber: um para cada município, para avaliar possíveis modificações de efeito entre a variável dependente e as independentes, e outro no qual o município de residência foi incluído como uma covariável do modelo. Os resultados apresentados se referem à estratégia considerada mais adequada.

\section{Resultados}

As mulheres residentes em Petrópolis representaram 58,3\% da amostra. Houve predominância de mulheres na faixa etária entre 20 e 34 anos $(70,0 \%)$, pretas e pardas $(65,0 \%)$, inseridas na classe social B e C $(69,0 \%)$, que viviam com companheiro $(73,0 \%)$ e que não tinham trabalho fora de casa $(58,0 \%)$. Quanto à ocorrência de morbidade, 2,0 e 19,0\% das mulheres referiram ter diabetes mellitus e hipertensão arterial, respectivamente (Tabela 1 ).

Pela avaliação antropométrica pré-gestacional, $63,0 \%$ das mulheres foram classificadas como eutróficas, $11,0 \%$ como baixo peso, $18,0 \%$ como sobrepeso e $9,0 \%$ como obesas. Em relação às características obstétricas, $55,3 \%$ das mulheres tinham experiência prévia de parto e $42,0 \%$ tiveram o pré-natal classificado na categoria mais que adequado. Quanto aos hábitos maternos, 12,0 e 6,0\% das mulheres referiram ter fumado e consumido bebida alcoólica durante a gestação, respectivamente.

Não houve modificação dos efeitos e os modelos foram semelhantes em ambos os municípios. Por este motivo foram realizadas as análises utilizando um modelo único a fim de melhorarmos a precisão dos dados. Na Tabela 2 são expostos os resultados da análise bivariada das variáveis independentes. Ser da classe social D/E foi o único fator associado ao ganho de peso abaixo do recomendado $(\mathrm{OR}=1,4 ;$ IC95\% 1,1-1,7).

Foi observado que a cada ano de estudo foi ampliada em $10 \%$ a chance de ganhar peso acima do recomendado $(\mathrm{OR}=1,1$; IC95\% 1,0-1,1). Do mesmo modo, a hipertensão arterial (OR $=2,5$; IC95\% 2,0-3,2), o sobrepeso $(\mathrm{OR}=2,8 ; \mathrm{IC} 95 \% 2,2-3,6)$ e a obesidade $(\mathrm{OR}=2,9$; IC95\% 2,0-4,2) no período pré-gestacional se mostraram associados ao ganho de peso acima do recomendado. Por outro lado, a adolescência ( $<20$ anos) $(\mathrm{OR}=0,7$; IC95\% $0,6-0,9)$ e o baixo peso pré-gestacional foram considerados fatores protetores para este desfecho $(\mathrm{OR}=0,6$; IC95\% 0,4-0,8).

No modelo multivariado nenhuma variável estudada permaneceu estatisticamente significativa para explicar o ganho de peso gestacional abaixo do recomendado. No entanto, mais anos de estudo (OR $=1,08$; IC95\% 1,0-1,1), a hipertensão arterial (OR=2,09; IC95\% 1,4-3,1), o sobrepeso (OR $=2,69$; IC95\% 1,8-3,9) e a obesidade $(\mathrm{OR}=2,54$; IC95\% 1,4-4,5) no período pré-gestacional permaneceram como variáveis explicativas do ganho de peso excessivo na gestação (Tabela 2 ).

\section{Discussão}

Mais recentemente, estudos sobre ganho de peso gestacional têm demonstrado uma tendência ao declínio do ganho de peso adequado em favor do aumento do ganho de peso excessivo ${ }^{6,8}$. O ganho de peso gestacional adequado, encontrado em menos de um terço das mulheres, foi similar ao reportado por Rebelo et al. ${ }^{21}$ em uma coorte de 104 gestantes recrutadas em uma unidade básica de saúde no município do Rio de Janeiro.

Também a proporção de mulheres com ganho de peso excessivo foi semelhante ao encontrado em uma coorte de 141 gestantes saudáveis atendidas em um serviço público de pré-natal em São Paulo ${ }^{12}$. No entanto, proporções menores (29\%) foram descritas por dois estudos brasileiros, um realizado em seis capitais, constituído por 3.082 gestantes da rede pública de saúde, e outro em uma coorte realizada no município do Rio de Janeiro. Esta diferença encontrada pode ser parcialmente justificada pela utilização de diferentes critérios para diagnosticar o estado nutricional pré-gestacional e o ganho de peso gestacional. O presente estudo utilizou a recomendação do IOM de $2009^{15}$, enquanto Nucci et al. ${ }^{6}$ e Kac e Velásquez-Meléndez ${ }^{22}$ utilizaram os critérios do IOM de $1990^{3}$. As diretrizes atuais ${ }^{15}$ diferem da precedente ${ }^{3}$ pelo fato do estado nutricional pré-gestacional, classificado pelo IMC, ser baseado nos pontos de corte da Organização Mundial de Saúde ${ }^{23}$. Além disso, esta nova recomendação delimitou uma faixa de ganho de peso para gestantes obesas ${ }^{23}$. A instituição dessas mudanças visou reduzir a retenção de peso pós-parto, doenças cardiovasculares, desordens metabólicas, dentre outros resultados para a mãe, além daquelas que interferem na saúde da criança ${ }^{15}$.

O estado nutricional pré-gestacional é um dos principais fatores associados ao ganho de peso durante a gravide $z^{24}$. A chance elevada de ganho excessivo de peso em gestantes com sobrepeso e obesidade pré-gestacional confirma os achados de duas coortes, uma com 141 gestantes saudáveis de um serviço público do município de São Paulo ${ }^{12}$ e outra com 667 gestantes acompanhadas até o parto na rede básica de saúde do Estado do Rio Grande do Sul ${ }^{25}$.

Estudo multicêntrico, conduzido nos Estados Unidos com 2.070 mulheres acompanhadas por 10 anos, mostrou que mulheres com sobrepeso no início 
Tabela 1. Características sociodemográficas, doenças crônicas, estado nutricional, adequação do pré-natal e hábitos de vida, segundo adequação do ganho de peso gestacional

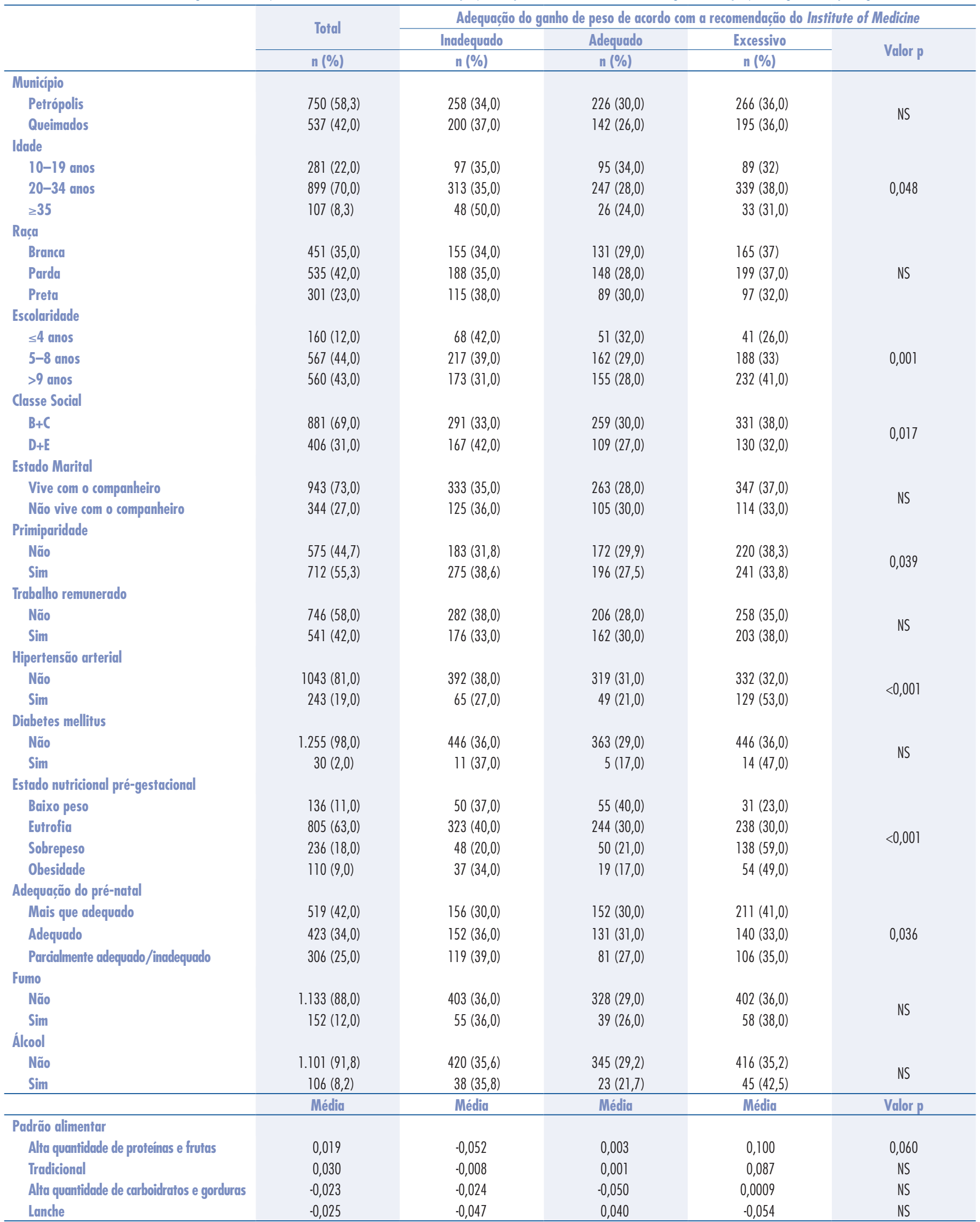

NS: não significativo. 


\begin{tabular}{|c|c|c|c|c|}
\hline & \multicolumn{4}{|c|}{ Adequação do ganho de peso de acordo com a recomendação do Institute of Medicine } \\
\hline & \multicolumn{2}{|c|}{ Análise bivariada } & \multicolumn{2}{|c|}{ Análise múltipla } \\
\hline & $\begin{array}{l}\text { Inadequado } \\
\text { OR (IC95\%) }\end{array}$ & $\begin{array}{c}\text { Excessivo } \\
\text { OR (IC95\%) }\end{array}$ & $\begin{array}{l}\text { Inadequado } \\
\text { OR (IC95\%) }\end{array}$ & $\begin{array}{l}\text { Excessivo } \\
\text { OR (IC95\%) }\end{array}$ \\
\hline \multicolumn{5}{|l|}{ Município } \\
\hline Queimados & $1,2(1,0-1,48)$ & $1,2(0,9-1,4)$ & - & - \\
\hline \multicolumn{5}{|l|}{ Idade } \\
\hline \multicolumn{5}{|l|}{ Raça } \\
\hline Parda & $1,1(0,9-1,3)$ & $1,1(0,9-1,3)$ & - & - \\
\hline Preta & $1,1(0,9-1,4)$ & $0,9(0,7-1,1)$ & - & - \\
\hline \multicolumn{5}{|l|}{ Escolaridade } \\
\hline Anos de estudo & $1,0(0,9-1,0)$ & $1,1(1,0-1,1)$ & $0,96(0,9-1,0)$ & $1,08(1,0-1,1)$ \\
\hline Não vive com o companheiro & $0,9(0,8-1,2)$ & $0,8(0,7-1,0)$ & - & - \\
\hline \multicolumn{5}{|l|}{ Primiparidade } \\
\hline Sim & $1,3(1,1-1,6)$ & $1,0(0,8-1,2)$ & - & - \\
\hline \multicolumn{5}{|l|}{ Trabalho remunerado } \\
\hline Sim & $0,8(0,7-1,0)$ & $1,0(0,8-1,2)$ & - & - \\
\hline \multicolumn{5}{|l|}{ Hipertensão arterial } \\
\hline Sim & $1,1(0,8-1,4)$ & $2,5(2,0-3,2)$ & $1,02(0,7-1,5)$ & $2,09(1,4-3,1)$ \\
\hline \multicolumn{5}{|l|}{ Diabetes mellitus } \\
\hline Sim & $1,8(0,9-3,6)$ & $2,3(1,2-4,4)$ & - & - \\
\hline \multicolumn{5}{|l|}{ Estado nutricional pré-gestacional } \\
\hline \multicolumn{5}{|l|}{ Fumo } \\
\hline Sim & $1,2(0,9-1,5)$ & $1,2(0,9-1,6)$ & - & - \\
\hline \multicolumn{5}{|l|}{ Álcool } \\
\hline Sim & $1,6(0,9-3,0)$ & $1,4(0,7-2,6)$ & - & - \\
\hline \multicolumn{5}{|l|}{ Padrão alimentar } \\
\hline Alta quantidade de proteínas e frutas & $0,9(0,9-1,0)$ & $1,1(1,0-1,2)$ & - & - \\
\hline Tradicional & $1,0(0,9-1,1)$ & $1,1(1,0-1,2)$ & - & - \\
\hline Alta quantidade de carboidratos e gorduras & $1,0(0,9-1,1)$ & $1,1(1,0-1,2)$ & - & - \\
\hline Lanche & $0,9(0,8-1,0)$ & $0,9(0,8-1,0)$ & - & - \\
\hline
\end{tabular}

da gestação ganharam mais peso em relação às eutróficas ${ }^{24}$. Nucci et al. ${ }^{6}$ também demonstraram ganho de peso acima do recomendado em aproximadamente metade das gestantes com sobrepeso prévio. O estilo de vida somado a fatores psicossociais podem estar associados ao maior ganho de peso entre mulheres com IMC pré-gestacional na faixa do sobrepeso e obesidade. Além disso, estas mulheres podem ter menos atitudes favoráveis para o alcance de ganho de peso adequado, tais como hábitos alimentares saudáveis e prática de atividade física regular, quando comparadas às mulheres com ganho de peso adequado ${ }^{8}$.

Nesta amostra, quão maior a escolaridade, em anos de estudo, maior a chance do ganho de peso acima do recomendado, tal como em estudo realizado em São Paulo (SP) ${ }^{7}$, em que gestantes com quatro anos ou mais de estudo apresentaram ganho de quase dois quilos a mais quando comparadas às demais. De acordo com este 
autor, a escolaridade foi considerada um marcador de acesso aos alimentos. Por outro lado, em Recife $(\mathrm{PE})^{8}$, a baixa escolaridade contribuiu para o ganho de peso excessivo. O nível de escolaridade reflete a situação socioeconômica, sendo assim, as gestantes com menor poder aquisitivo priorizam o consumo de alimentos mais calóricos (ricos em gorduras, particularmente de origem animal, açúcar e alimentos refinados, em detrimento aos carboidratos complexos e fibras) por terem menor custo. Cabe destacar que o nível de escolaridade foi avaliado de forma contínua nas análises bi e multivariada e que houve predomínio de mulheres com até 9 anos de estudo (56\%). Além disso, trata-se de mulheres usuárias do SUS, em geral, com baixo poder aquisitivo.

Devido à baixa prevalência de diabetes mellitus nesta amostra não foi possível observar associação entre esta doença e a inadequação do ganho de peso gestacional. No entanto, em direção contrária, foi demonstrado que o ganho de peso excessivo é um fator de risco para o diabetes mellitus ${ }^{26}$. Por outro lado, mulheres com diabetes iniciam a gestação com IMC mais elevado, porém ganham menos peso ${ }^{1}$. Acredita-se que isso se deva à promoção da reeducação alimentar realizada nas consultas de pré-natal pelos profissionais de saúde ${ }^{27}$.

No presente estudo, as mulheres que relataram hipertensão arterial tiveram mais chance de ganhar peso acima do recomendado. Independentemente da direção da associação entre estas variáveis, o IMC elevado no período pré-gestacional bem como o ganho de peso excessivo elevam os riscos para a hipertensão arterial ${ }^{27}$.

Apesar da associação entre classe social D/E e ganho de peso abaixo do recomendado, houve perda da significância desse resultado na análise multivariada. As características sociodemográficas também podem contribuir para um acesso desigual aos serviços de saúde, sendo assim, mulheres de baixa renda são mais desprovidas da assistência no pré-natal e puerpério e, consequentemente, de informações básicas necessárias ao melhor desfecho reprodutivo.

O resultado de um estudo conduzido no Canadá divergiu do presente estudo, uma vez que demonstrou que as mulheres com baixo status socioeconômico ganharam mais peso em relação às demais. Isso, provavelmente, se deve à extrema diferença social e econômica deste país em relação à nossa amostra, no que diz respeito ao nível educacional, renda e acesso a serviços de saúde.

Considera-se que os parâmetros empregados para avaliar classe social nesse estudo precisam ser revistos, pois com a melhoria das condições de vida da população brasileira nos últimos $\operatorname{anos}^{28}$, esses já não discriminam tanto o acesso a bens de consumo. Dessa forma, acredita-se que as mulheres classificadas nas categorias D e E, representam aquelas em extrema vulnerabilidade social.

Ainda que os dois municípios se diferenciem, especialmente no que diz respeito ao aspecto socioeconômico e de capital social ${ }^{18}$, os determinantes associados à inadequação do ganho de peso na gestação foram os mesmos (dados não mostrados). Ou seja, mesmo em Petrópolis onde as mulheres têm melhores condições de vida, os fatores associados ao desfecho em questão se restringiram às características individuais das gestantes, como idade, escolaridade, estado de saúde e nutricional.

Algumas limitações desse estudo merecem ser discutidas. As informações sobre o peso pré-gestacional e peso ao final da gestação foram obtidas pelo relato da mulher. Priorizou-se a utilização desta informação, pois apenas uma parcela das mulheres possuía o cartão de pré-natal com esse dado preenchido; sendo a grande maioria do município de Petrópolis. De acordo com Silveira et al. ${ }^{29}$, existe alta relação entre os valores mensurados de peso e aqueles autoinformados; mas, alguns desses mostraram tendência a subestimativa. Este resultado é confirmado pelo estudo realizado com 150 gestantes atendidas em dois hospitais do município do Rio de Janeiro o qual demonstrou elevada concordância/correlação entre as variáveis medidas e referidas do peso pré-gestacional e do peso ao final da gravidez utilizando o coeficiente de correlação de Pearson e intraclasse ${ }^{30}$.

Embora o ganho ponderal insuficiente persista como um problema de saúde, o ganho de peso excessivo se configura como uma questão que precisa de atenção imediata nos serviços de saúde, considerando-se as consequências adversas advindas desse fato para o binômio mãe/filho.

A expressiva quantidade de mulheres com inadequação do ganho de peso gestacional encontrada neste estudo demanda que a orientação nutricional se inicie desde a puericultura com vistas a promover mudanças no estilo de vida ${ }^{8}$ e reverter esse padrão alimentar adotado também pela população brasileira que prioriza o consumo de produtos ricos em açúcar refinado, gorduras saturadas, refrigerantes e alimentos processados, em substituição a base alimentar do brasileiro ${ }^{11}$.

\section{Agradecimentos}

Ao Conselho Nacional de Desenvolvimento Científico e Tecnológico (CNPq) e à Fundação de Amparo à Pesquisa do Estado do Rio de Janeiro (FAPERJ). 
1. Salmenhaara M, Uusitalo L, Uusitalo U, Kronberg-Kippila C, Sinkko $\mathrm{H}$, Ahonen $\mathrm{S}$, et al. Diet and weight gain characteristics of pregnant women with gestational diabetes. Eur J Clin Nutr. 2010;64(12): 1433-40.

2. Kowal C, Kuk J, Tamim H. Characteristics of weight gain in pregnancy among Canadian women. Matern Child Health J. 2012; 16(3):668-76.

3. National Academy of Science. Institute of Medicine. Nutrition during pregnancy: Part l: weight gain, Part II: nutrient supplements. Washington (DC): National Academy Press; 1990.

4. Padilha PC, Saunders C, Machado RCM, Silva CL, Bull A, Sally $E O F$, et al. [Association between pre-gestational nutritional status and prediction of the risk of adverse pregnancy outcome]. Rev Bras Ginecol Obstet. 2007;29(10):51 1-8. Portuguese.

5. Nomura RMY, Paiva LV, Costa VN, Liao AW, Zugaib M. [Influence of maternal nutritional status, weight gain and energy intake on fetal growth in high-risk pregnancies]. Rev Bras Ginecol Obstet. 2012;34(3):107-12. Portuguese.

6. Nucci LB, Duncan BB, Mengue SS, Branchtein L, Schmidt $\mathrm{MI}$, Fleck ET. Assessment of weight gain during pregnancy in general prenatal care services in Brazil. Cad Saúde Pública. $2001 ; 17(6): 1367-74$.

7. Konno SC, Benicio MHDA, Barros AJD. Fatores associados à evolução ponderal de gestantes: uma análise multinível. Rev Saúde Pública. 2007;41(6):995-1002.

8. Andreto LM, Souza Al, Figueiroa JN, Cabral-Filho JE. Fatores associados ao ganho ponderal excessivo em gestantes atendidas em um serviço público de pré-natal na cidade de Recife, Pernambuco, Brasil. Cad Saúde Pública. 2006;22(1 1):2401-9.

9. Phelan S, Phipps MG, Abrams B, Darroch F, Schaffner A, Wing RR. Randomized trial of a behavioral intervention to prevent excessive gestational weight gain: the fit for delivery study. Am J Clin Nutr. 2011 ; 93(4):772-9.

10. Barros DC, Saunders C, Leal MC. Avaliação nutricional antropométrica de gestantes brasileiras: uma revisão sistemática. Rev Bras Saúde Matern Infant. 2008;8(4):363-76.

11. Vítolo MR, Bueno MSF, Gama CM. [Impact of a dietary counseling program on the gain weight speed of pregnant women attended in a primary care service]. Rev Bras Ginecol Obstet. $2011 ; 33(1): 13$ 9. Portuguese.

12. Stulbach TE, Benício MHDA, Andreazza R, Kono S. Determinantes do ganho ponderal excessivo durante a gestação em serviço público de pré-natal de baixo risco. Rev Bras Epidemiol. 2007;10(1):99-108.

13. Rodrigues PL, Lacerda EM, Schlussel MM, Spyrides $M H$, Kac $G$. Determinants of weight gain in pregnant women attending a public prenatal care facility in Rio de Janeiro, Brazil: a prospective study, 2005-2007. Cad Saúde Pública. 2008;24 Suppl 2:S272-84.

14. Brasil. Ministério da Saúde. Secretaria de Atenção Básica [Internet]. Cadastro Nacional de Estabelecimentos de Saúde [cited 2006 Jun 12]. Available from: http://cnes.datasus.gov. br/Mod_Ind_Unidade.asp
15. Rasmussen KM, Yaktine AL, editors; Committee to Reexamine IOM Pregnancy Weight Guidelines, Food and Nutrition Board and Board on Children, Youth, and Families. Washington (DC): National Academy Press; 2009.

16. Instituto Brasileiro de Geografia e Estatistica (IBGE) [Internet]. Rio de Janeiro: IBGE; 2008 [cited 2009 Jul 12]. Available from: http://www.ibge.gov.br

17. Associação Brasileira de Empresas de Pesquisa (ABEP). Adoção do CCEB 2008: Critério de Classificação Econômica Brasil. São Paulo: ABEP; 2008.

18. Leal MC, Pereira AP, Lamarca GA, Vettore MV. The relationship between social capital, social support and the adequate use of prenatal care. Cad Saúde Pública. 2011 ;27 Suppl 2:S237-53.

19. Godzik B, Manning WJ, Sichieri R, Everhart JE. Validity of a Brazilian food frequency questionnaire against dietary recalls and estimated energy intake. Nutr Res. 1998;18(10): 1649-59.

20. Conselho Nacional de Saúde. Resolução n 196/96 sobre pesquisa envolvendo seres humanos. Bioética. 1996;4(2 Supl): 15-25.

21. Rebelo F, Castro MBT, Dutra CL, Schlussel MM, Kac G. Fatores associados à retenção de peso pós-parto em uma coorte de mulheres, 2005-2007. Rev Bras Saúde Matern Infant. 2010;10(2):219-27.

22. Kac G, Velásquez-Meléndez G. Ganho de peso gestacional macrossomia em uma coorte de mães e filhos. J Pediatr (Rio J). 2005;81(1):47-53.

23. Subhadra B, Grinson-George. Algal biorefinery-based industry: an approach to address fuel and food insecurity for a carbon-smart world. J Sci Food Agric. 2011 ;91 (1):2-13.

24. Gunderson EP, Murtaugh MA, Lewis CE, Quesenberry CP, West DS, Sidney $S$. Excess gains in weight and waist circumference associated with childbearing: the coronary artery risk development in young adults study (CARDIA). Int J Obes Relat Metab Disord 2004;28(4):525-35.

25. Drehmer M, Camey S, Schmidt MI, Olinto MT, Giacomello A, Buss $C$, et al. Socioeconomic, demographic and nutritional factors associated with maternal weight gain in general practices in Southern Brazil. Cad Saúde Pública. 2010;26(5):1024-34.

26. Gibson KS, Waters TP, Catalano PM. Maternal weight gain in women who develop gestational diabetes mellitus. Obstet Gynecol. 2012;1 19(3):560-5.

27. Tabatabaei M. Gestational weight gain, prepregnancy body mass index related to pregnancy outcomes in KAZERUN, FARS, IRAN. J Prenat Med. 201 1;5(2):35-40.

28. Furtado BA. Índice de vulnerabilidade das famílias: atualização (2003-2009) e recortes geográficos. Brasília (DC): IPEA; 2012.

29. Silveira EA, Araújo CL, Gigante DP, Barros AJD, Lima MS. Validação do peso e altura referidos para o diagnóstico do estado nutricional em uma população de adultos no Sul do Brasil. Cad Saúde Pública. 2005;21(1):235-45.

30. Oliveira AF, Gadelha AM, Leal MC, Szwarcwald CL. Estudo da validação das informações de peso e estatura em gestantes atendidas em maternidades municipais no Rio de Janeiro, Brasil. Cad Saúde Pública. 2004;20 Suppl1:S92-100. 